\title{
Long-term radiation therapy-related risk of second primary malignancies in patients with lung cancer
}

\author{
Chang Han", Yijun Wu", Kai Kang", Zhile Wang, Zhikai Liu*, Fuquan Zhang* \\ Department of Radiation Oncology, Peking Union Medical College Hospital, Chinese Academy of Medical Sciences \& Peking Union Medical \\ College, Beijing, China \\ Contributions: (I) Conception and design: F Zhang, C Han; (II) Administrative support: Z Liu, F Zhang; (III) Provision of study materials or patients: \\ C Han; (IV) Collection and assembly of data: Z Wang, C Han; (V) Data analysis and interpretation: K Kang, Y Wu; (VI) Manuscript writing: All \\ authors; (VII) Final approval of manuscript: All authors. \\ "These authors contributed equally as the first authors. \\ *These authors contributed equally as the corresponding authors. \\ Correspondence to: Fuquan Zhang, MD; Zhikai Liu. Department of Radiation Oncology, Peking Union Medical College Hospital, Chinese Academy \\ of Medical Sciences \& Peking Union Medical College, Beijing 100730, China. Email: zhangfq_pumc@163.com; liuzk2009@126.com.
}

Background: With the improvement of cancer therapy, a second primary malignancy (SPM) occurs more commonly among cancer survivors. At present, it remains unclear whether the radiation therapy for the initial lung cancer will increase the risk of developing a SPM. This study aims to investigate the long-term risk of a SPM attributable to the radiation therapy in patients with the initial lung cancer.

Methods: Patients initially diagnosed with lung cancer between January 1975 and November 2011 were identified from the Surveillance, Epidemiology, and End Results (SEER) database. SPM was defined as the occurrence of a second cancer at least five years after the diagnosis of the initial lung cancer. Age- and propensity score matching (PSM)-adjusted competing risk analyses were performed to compare the risk of SPM.

Results: Of 47,911 patients, 9,162 (19.1\%) underwent radiotherapy for the initial lung cancer. The PSMadjusted competing risk analyses showed that radiation therapy was associated with a lower overall risk of SPM (HR: 0.89, 95\% CI: 0.84-0.94, P<0.001). Specifically, the risk of second primary melanoma (HR: 0.49, 95\% CI: 0.29-0.81, P=0.006), second primary female breast cancer (HR: 0.65, 95\% CI: 0.50-0.85, P=0.001), second primary prostate cancer (HR: 0.69, 95\% CI: 0.58-0.84, $\mathrm{P}<0.001)$ and second primary thyroid cancer (HR: 0.23 , 95\% CI: $0.07-0.77, \mathrm{P}=0.017$ ) was found to decrease, while the risk for second primary esophageal cancer dramatically increased (HR: $1.76,95 \%$ CI: 1.26-2.45, $\mathrm{P}<0.001$ ).

Conclusions: In patients who received radiotherapy for the initial lung cancer, the risk decreased for second primary melanoma as well as for second primary cancers of female breast, prostate and thyroid gland but increased for second primary cancer of esophagus. On the whole, radiation therapy for initial lung cancer may not increase the overall risk of SPM.

Keywords: Second malignancy; radiotherapy; lung cancer; competing risk regression; propensity score matching analysis

Submitted Jun 02, 2021. Accepted for publication Sep 08, 2021.

doi: $10.21037 /$ jtd-21-915

View this article at: https://dx.doi.org/10.21037/jtd-21-915 


\section{Introduction}

Lung cancer is the cause of the highest cancer-related mortality in recent years (1). Multiple therapeutic modalities have been applied to treat lung cancer, including surgery, radiation therapy, systemic treatment (targeted therapy, chemotherapy and immunotherapy), interventional methods and palliative care. The rapid development of radiotherapy technologies improved curative effects with more indications and fewer side effects for tumor therapy (2). For lung cancer patients, radiation therapy can be adopted in all stages. It was estimated that as high as $77 \%$ of lung cancer patients had an indication for radiation treatment (3). Although radiotherapy demonstrated encouraging overall survival and local control rates when compared to surgery $(4,5)$, its effects on long-term outcome in terms of survival, complications and risk of other diseases are less clear.

As the number of cancer survivors grows, second primary malignancy (SPM) has become an increasingly significant issue in cancer surveillance, diagnosis and management. It has been reported that cancer survivors would be at a higher risk of developing a second primary cancer (6). In a Surveillance, Epidemiology, and End Results (SEER) database analysis by Thakur et al., 2.95\% of lung cancer patients developed a second lung cancer with a risk ratio of 3.98 (Observed/Expected: 4,622/1,161.6) (7). Additionally, primary tumors of other organs may also occur more frequently in patients who previously suffered lung cancer (8). The cause of SPM may be attributable to a variety of potential factors, such as lifestyle, genetic background and the effect of treatments for the initial primary cancer. Berrington de Gonzalez et al. reported that radiotherapy could increase the relative risk of developing SPM by $6 \%$ among former non-small cell lung cancer (NSCLC) patients who have survived more than a year (9). However, as the predominant competing event with death, whether the long-term risk of SPM is associated with radiotherapy remains unclear. Hence, among lung cancer patients, the present study performed competing risk analysis to investigate the influence of radiation therapy on the long-term risk of developing SPM.

We present the following article in accordance with the STROBE reporting checklist (available at https://dx.doi. org/10.21037/jtd-21-915).

\section{Methods}

\section{Data source}

The SEER program (http://seer.cancer.gov/) launched by the National Cancer Institute of the United States is a population-based cancer database that collects information on clinical demographics, epidemiology, treatment and follow-up. The present study was limited to 9 registries in SEER, including Atlanta, Connecticut, Detroit, Hawaii, Iowa, New Mexico, San Francisco-Oakland, SeattlePuget Sound, and Utah. All patients' information in the SEER public database was anonymized. Thus, no informed consent was required, and this research was exempted by the Ethics Committee of Peking Union Medical College Hospital. The study was conducted in accordance with the Declaration of Helsinki (as revised in 2013).

\section{Patient coborts}

Patients aged 20 years or older who were diagnosed with primary lung cancer between January 1975 and November 2011 were extracted from the SEER database. The last follow-up was updated in November 2016. The initial inclusion strategy was set as follows: (I) primary site: lung and bronchus (ICD-O-3/WHO 2008, International Classification of Diseases for Oncology, Third Edition); (II) age at diagnosis of the first primary lung cancer (FPLC): 20 years or older; and (III) date of diagnosis: January 1975 to November 2011. Additionally, patients who met one of the following criteria were excluded: (I) lung cancer not as the initial cancer; (II) death or development of a second cancer within 5 years after the diagnosis of FPLC; and (III) incomplete survival and follow-up information. Only patients diagnosed before November 2011 were considered in the analysis to ensure that all included patients were followed up for at least 5 years, a lag that can be long enough to distinguish SPM from late metastases of FPLC and to expose the effect of radiation therapy on SPM induction (10).

\section{Study parameters and classifications}

The demographic and treatment data of patients extracted from the SEER database included diagnosis year of 
(1975-1984, 1985-1994, 1995-2004 and 2005-2011), age (20-39, 40-64 and $\geq 65$ years), sex (male and female), race [white, black, others (American Indian/AK Native, Asian/ Pacific Islander) and unknown], primary location (upper lobe, middle lobe, lower lobe, main bronchus, overlapping lesion and unknown), histologic type (adenocarcinoma: code 8140, 8250-8253, 8255, 8260, 8323, 8480-8481, 8550, 8560, 8570, 8574; squamous cell carcinoma, SCC: 8070-8073, 8083; small cell lung cancer, SCLC: 8041, 8045; and other NSCLC); SEER cancer stage (localized, regional, distant and unknown); surgery for FPLC (yes and no); chemotherapy for FPLC (yes and no/unknown); and radiotherapy for FPLC (yes and no).

According to ICD-O-3/WHO 2008, site details of SPM were also extracted. It is conceivable that other organs have been exposed to radiation during the radiotherapy for FPLC, and that the dosage depends on the distance between the organ and the lung. The radiation doses at various distances can be estimated from standard radiotherapy protocols. Therefore, based on the distances between the organ and the border of the radiation field of the lung, SPM sites were classified into three dose groups: high-dose (<3 cm, >5 gray), medium-dose $(3-10 \mathrm{~cm}, 1-5$ gray) and low-dose ( $\geq 10 \mathrm{~cm}, \leq 1$ gray) $(9,11)$.

\section{Statistical analysis}

SEER * Stat software version 8.3.6 was used to extract patient information from the SEER database. All statistical analyses were performed using SPSS 25.0 (Chicago, IL, USA) and R software version 3.6.3 (http://www.r-project.org). A P value $<0.05$ (two-sided) was considered statistically significant. Clinical characteristics of FPLC were summarized using counts and percentages, and differences were compared by Pearson's chi square test. For further analysis, potential predictors of radiotherapy were assessed by multivariate logistic regression.

To identify the association between the risk of SPM and radiation therapy, competing risk analyses and the Fine-Gray test were employed $(12,13)$. The conventional statistical methods for survival data regarded individuals who died before the interest event (developing SPM in our study), who did not have the interest event until the end of the follow-up and who were lost to follow-up as censored data, which may lead to risk estimation bias (12). Thus, using the "cmprsk" R package (14), age-adjusted competing risk regression analyses were performed. For calculating the whole risk of SPM, death and SPM were regarded as competing events, while for calculating the risk of one single site of second cancer, other malignancies and death were regarded as competing events. In addition, considering the confounding effects of demographic and therapeutic data, competing risk regression was further adjusted by propensity score matching (PSM) to ensure that there were consistent distributions of characteristics between patients with and without radiation therapy. During the matching procedure, each patient in both groups was matched to possible patients in the other group, and all unmatched patients were excluded from PSM-adjusted competing risk regression analyses.

\section{Results}

\section{Patient characteristics}

After extraction and selection, a total of 47,911 lung cancer patients who met our inclusion criteria were identified, including 24,093 (50.3\%) males and 23,818 (49.7\%) females (Table 1). Among them, 12,834 (26.8\%) individuals received radiation therapy for lung cancer. The median follow-up time was 9.3 [interquartile range (IQR), 6.8-13.8] years. The median age of all patients was 64 (IQR, 57-71) years. Most patients $(83.8 \%, 40,156 / 47,911)$ underwent surgical resection for lung cancer, while $21.5 \%(10,311 / 47,911)$ of them received chemotherapy before or after surgery. Table 1 shows the comparisons of characteristics between two groups by univariate analyses and multivariate logistic regression. Since 1975, the number of patients receiving radiotherapy has increased over time. Patients in middle age (40-64 vs. 20-39 years; odds ratio, OR: 1.37, 95\% CI: $1.16-$ 1.62, $\mathrm{P}<0.001$ ), male gender (vs. female; OR: 1.06, 95\% CI: 1.01-1.12, $\mathrm{P}=0.016)$, SCC (vs. adenocarcinoma; OR: 1.73, 95\% CI: $1.62-1.84, \mathrm{P}<0.001)$, SCLC (vs. adenocarcinoma; OR: 2.09, 95\% CI: 1.85-2.36, $\mathrm{P}<0.001$ ), other NSCLC (vs. adenocarcinoma; OR: 1.35, 95\% CI: 1.27-1.44, $\mathrm{P}<0.001)$, regional (vs. localized; OR: 4.62, 95\% CI: 4.30-4.96, $\mathrm{P}<0.001$ ) and distant (vs. localized; OR: 2.26, 95\% CI: 2.04-2.51, $\mathrm{P}<0.001)$ SEER stages, surgical resection (vs. no surgery; OR: 6.88, 95\% CI: 6.36-7.43, $\mathrm{P}<0.001)$ and no chemotherapy (vs. chemotherapy; OR: 5.87, 95\% CI: $5.50-6.26, \mathrm{P}<0.001)$ were more likely to undergo radiation therapy.

\section{Age-adjusted competing risk analysis}

Of all 47,911 patients, SPM was observed in 9,162 (19.1\%) 
Table 1 Univariate and multivariate analyses of patients' characteristics for the prediction of radiation therapy

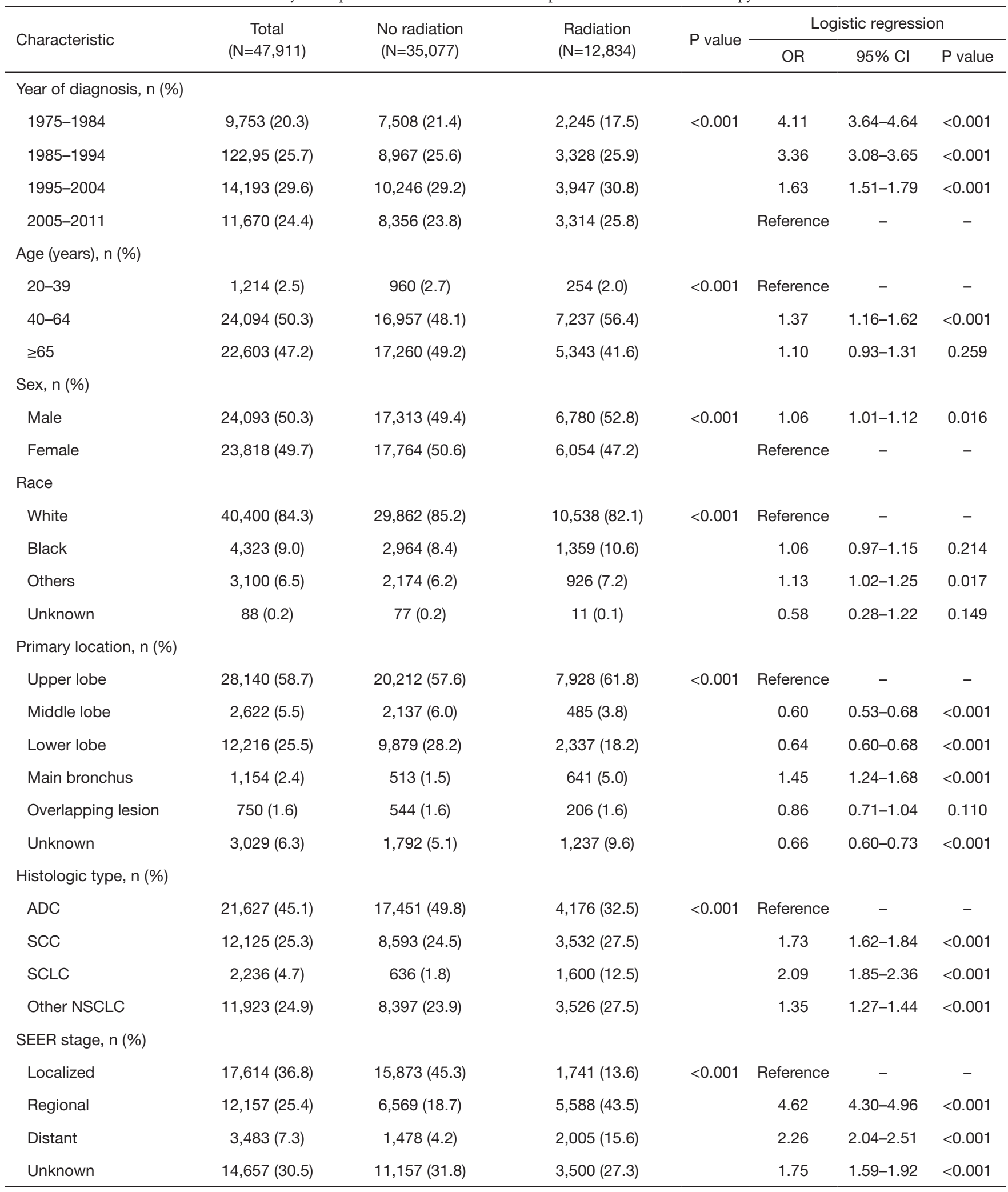

Table 1 (continued) 
Table 1 (continued)

\begin{tabular}{|c|c|c|c|c|c|c|c|}
\hline Characteristic & $\begin{array}{c}\text { Total } \\
(\mathrm{N}=47,911)\end{array}$ & $\begin{array}{l}\text { No radiation } \\
(\mathrm{N}=35,077)\end{array}$ & $\begin{array}{l}\text { Radiation } \\
(\mathrm{N}=12,834)\end{array}$ & $P$ value & \multicolumn{3}{|c|}{ Logistic regression } \\
\hline \multicolumn{8}{|l|}{ Surgery, n (\%) } \\
\hline Yes & $40,156(83.8)$ & 32,335 (92.2) & $7,821(60.9)$ & $<0.001$ & 6.88 & $6.36-7.43$ & $<0.001$ \\
\hline No & 7,755 (16.2) & $2,742(7.8)$ & $5,013(39.1)$ & & Reference & - & - \\
\hline Yes & $10,311(21.5)$ & $3,606(10.3)$ & 6,7605 (52.2) & $<0.001$ & Reference & - & - \\
\hline No/unknown & $37,600(78.5)$ & 31,471 (89.7) & $6,129(47.8)$ & & 5.87 & $5.50-6.26$ & $<0.001$ \\
\hline
\end{tabular}

OR, odds ratio; ADC, adenocarcinoma; SCC, squamous cell carcinoma; SCLC, small cell lung cancer; NSCLC, non-small cell lung cancer.

cases (Table 2). The most common sites of SPM included the lung and bronchus $(\mathrm{N}=3,908,42.7 \%)$; prostate $(\mathrm{N}=909$, 9.9\%); colon, rectum and anus ( $\mathrm{N}=750,8.2 \%)$; female breast $(\mathrm{N}=607,6.6 \%)$; bladder $(\mathrm{N}=503,5.5 \%)$; hematological system $(\mathrm{N}=498,5.4 \%)$; oral cavity and pharynx $(\mathrm{N}=237$, $2.6 \%)$; pancreas ( $\mathrm{N}=200,2.2 \%)$; esophagus $(\mathrm{N}=198,2.2 \%)$; larynx (N=173, 1.9\%); female genital system ( $\mathrm{N}=161,1.8 \%)$; and skin melanoma (N=157, 1.7\%; Figure 1).

Through age-adjusted competing risk analyses, radiation therapy was significantly associated with a lower risk of developing SPM [hazard ratio (HR): 0.87, 95\% CI: $0.83-$ 0.91, $\mathrm{P}<0.001$; Table 2 and Figure 2], while it was associated with a higher risk of death (HR: 1.47, 95\% CI: 1.43-1.51, $\mathrm{P}<0.001)$. Figure 3 depicts the cumulative incidence for the most common second malignancies in the age-adjusted competing risk analyses. Compared with those who did not receive radiotherapy, lung cancer patients in the radiation group were much more likely to suffer a second primary esophageal cancer (HR: 1.87, 95\% CI: 1.41-2.48, $\mathrm{P}<0.001$ ). However, radiation therapy was significantly associated with a lower incidence of second malignancies of the pancreas (HR: 0.63, 95\% CI: 0.44-0.89, $\mathrm{P}=0.010)$, melanoma (HR: 0.60, 95\% CI: 0.40-0.91, $\mathrm{P}=0.015$ ), female breast (HR: 0.67, 95\% CI: 0.54-0.82, $\mathrm{P}<0.001$ ), prostate (HR: 0.60, 95\% CI: 0.51-0.72, $\mathrm{P}<0.001$ ), bladder (HR: 0.75, 95\% CI: 0.61-0.93, $\mathrm{P}=0.009$ ) and thyroid (HR: 0.43 , 95\% CI: $0.20-0.90$, $\mathrm{P}=0.026$ ).

\section{PSM-adjusted competing risk analysis}

Furthermore, using the nearest neighbor matching algorithm with a caliper of 0.1 , patient characteristics were adjusted by PSM for competing risk analyses, including year of diagnosis, age, sex, race, primary location, histologic type, SEER stage, surgery and chemotherapy (the after-matching outcome was examined by histogram of standardized differences, dot plot of standardized mean differences and linear plot of individual differences). After matching at a ratio of $1: 2$ by dividing patients into two groups with and without radiotherapy, a total of 27,966 lung cancer patients (radiation vs. no radiation: 9,180 vs. 18,816 patients) were further analyzed (Table 2). Similarly, radiation therapy was still significantly associated with a lower risk of developing SPM (HR: 0.89, 95\% CI: 0.84-0.94, P<0.001). Among patients in the radiation group, the risk of second primary esophageal cancer greatly increased (HR: 1.76, 95\% CI: $1.26-2.45, \mathrm{P}<0.001)$, while the risk of second malignancies of melanoma (HR: 0.49, 95\% CI: 0.29-0.81, $\mathrm{P}=0.006$ ) and thyroid cancer (HR: $0.23,95 \%$ CI: $0.07-0.77$, $\mathrm{P}=0.017$ ) significantly decreased. Additionally, radiation therapy might also indicate a lower risk of second primary breast cancer in females (HR: 0.65, 95\% CI: 0.50-0.85, $\mathrm{P}=0.001$ ) and prostate cancer (HR: $0.69,95 \%$ CI: $0.58-0.84$, $\mathrm{P}<0.001)$ in males.

The age- and PSM-adjusted competing risk analyses for SPM in subgroups divided by patients' clinical and therapeutic characteristics are described in Table S1.

\section{Effect of radiation dose on SPM}

Based on standard radiotherapy dose protocols, the organs with a risk of developing SPM were categorized into dose groups: low, medium, high and inseparable (Figure 4 and Table S2). Although a lower risk of SPM was observed in patients who received radiotherapy than in those who did not, protection from SPM attributable to radiation therapy 
Table 2 The risk for second primary malignancies after radiation therapy for initial lung cancer

\begin{tabular}{|c|c|c|c|c|c|c|c|c|}
\hline Second malignancy & \multicolumn{4}{|c|}{ Age-adjusted competing risk regression } & \multicolumn{4}{|c|}{ PSM-adjusted competing risk regression } \\
\hline All patients & 47,911 & 9,162 & $0.87(0.83-0.91)$ & $<0.001$ & 27,996 & 5,428 & $0.89(0.84-0.94)$ & $<0.001$ \\
\hline Oral cavity and pharynx & 47,911 & 237 & $0.82(0.61-1.11)$ & 0.21 & 27,996 & 146 & $0.85(0.59-1.22)$ & 0.37 \\
\hline \multicolumn{9}{|l|}{ Digestive system } \\
\hline Stomach & 47,911 & 130 & $1.24(0.86-1.80)$ & 0.25 & 27,996 & 83 & $1.02(0.64-1.61)$ & 0.94 \\
\hline Colon, rectum and anus & 47,911 & 750 & $0.88(0.74-1.04)$ & 0.12 & 27,996 & 490 & $0.83(0.68-1.01)$ & 0.058 \\
\hline Hepatobiliary system & 47,911 & 114 & $0.90(0.59-1.37)$ & 0.62 & 27,996 & 63 & $0.78(0.45-1.37)$ & 0.39 \\
\hline Pancreas & 47,911 & 200 & $0.63(0.44-0.89)$ & 0.010 & 27,996 & 119 & $0.71(0.47-1.08)$ & 0.11 \\
\hline Lung and bronchus & 47,911 & 3,908 & $0.95(0.88-1.02)$ & 0.14 & 27,996 & 2,195 & $1.04(0.88-1.05)$ & 0.41 \\
\hline Larynx & 47,911 & 173 & $1.11(0.80-1.55)$ & 0.53 & 27,996 & 121 & $0.97(0.66-1.42)$ & 0.86 \\
\hline Others & 47,911 & 26 & - & - & 27,996 & - & - & - \\
\hline \multicolumn{9}{|c|}{ Skin excluding basal and squamous } \\
\hline Melanoma of the skin & 47,911 & 157 & $0.60(0.40-0.91)$ & 0.015 & 27,996 & 96 & $0.49(0.29-0.81)$ & 0.006 \\
\hline Other non-epithelial skin & 47,911 & 28 & - & - & - & - & - & - \\
\hline \multicolumn{9}{|l|}{ Breast } \\
\hline Female breast & 23,818 & 607 & $0.67(0.54-0.82)$ & $<0.001$ & 12,655 & 303 & $0.65(0.50-0.85)$ & 0.001 \\
\hline Others & 24,093 & 7 & - & - & 15,341 & - & - & - \\
\hline \multicolumn{9}{|l|}{ Urinary system } \\
\hline Bladder & 47,911 & 503 & $0.75(0.61-0.93)$ & 0.009 & 27,996 & 307 & $0.83(0.65-1.07)$ & 0.15 \\
\hline Kidney & 47,911 & 153 & $1.15(0.81-1.63)$ & 0.45 & 27,996 & 93 & $1.40(0.92-2.12)$ & 0.11 \\
\hline Others & 47,911 & 32 & - & - & 27,996 & - & - & - \\
\hline Nervous system & 47,911 & 46 & $0.67(0.32-1.40)$ & 0.29 & 27,996 & 16 & $0.96(0.33-2.77)$ & 0.94 \\
\hline Endocrine system & 47,911 & & & & & & & \\
\hline Thyroid & 47,911 & 58 & $0.43(0.20-0.90)$ & 0.026 & 27,996 & 30 & $0.23(0.07-0.77)$ & 0.017 \\
\hline Others & 47,911 & 8 & - & - & 27,996 & & & \\
\hline Hematological system & 47,911 & 498 & $0.87(0.71-1.07)$ & 0.19 & 27,996 & 302 & $0.94(0.74-1.20)$ & 0.62 \\
\hline Miscellaneous & 47,911 & 155 & $0.91(0.63-1.31)$ & 0.6 & 27,996 & 95 & $1.03(0.67-1.57)$ & 0.91 \\
\hline Other tumors & 47,911 & 57 & $0.86(0.63-1.18)$ & 0.36 & 27,996 & 121 & $0.76(0.51-1.14)$ & 0.18 \\
\hline
\end{tabular}

HR, hazards ratio. 


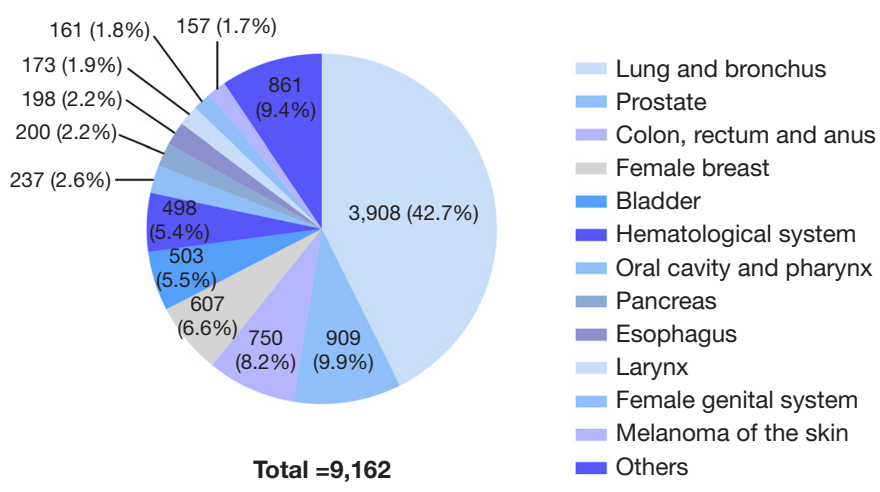

Figure 1 Sites of second primary malignancies after radiation therapy for initial lung cancer.

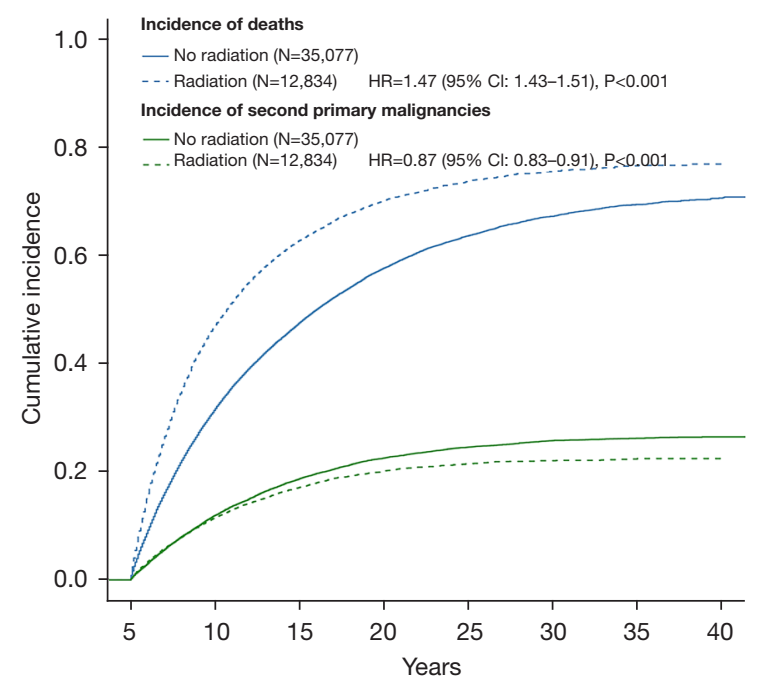

Figure 2 Cumulative incidence for the overall risk of second primary malignancies and death by age-adjusted competing risk analyses.

waned in the second cancer sites with higher radiation doses (Figure 4).

\section{Discussion}

The survival rate for many cancers has improved in recent decades because of encouraging advances in cancer treatments and supportive medical care. Unfortunately, prolonged survival in patients with previously treated malignancies is associated with an increased risk of developing SPM. Thus, more studies that focus on the incidence of SPM among cancer survivors are urgently needed for more precise surveillance and better management.
With the rapid deployment of low-dose computed tomography as a screening tool in high-risk populations, an increasing number of lung cancers are diagnosed at early stages (15). The 5 -year survival of stage IA NSCLC patients could be as high as $90 \%$ (16). In addition to the extended survival of lung cancer patients, there were other potential factors responsible for the increased risk of SPM, such as treatment modalities for the initial lung cancer. Previous studies have reported various findings regarding whether thoracic radiation therapy could increase the risk of SPM in the initial breast cancer patients (17-19), but very few have focused on those who received radiation therapy for the initial lung cancer.

In the present study, using the large amount of clinical data from the SEER database, we first investigated the longterm effects of radiation therapy on the development of SPM in patients with initial lung cancer. SPM was defined as a second primary cancer with more than 5 years of latency after the initial lung cancer, so that there was an at least 5 years have elapsed between radiation exposure and cancer induction (10). This restrictive criterion could exclude relapses and metastases of the initial lung cancer as much as possible and decrease the follow-up bias between different treatments. Patients who receive radical radiotherapy for lung cancer may be followed up more closely. Furthermore, by filtering the data with the 5 -year latency, we also tried to exclude patients who received radiation therapy for metastatic sites of lung cancer because the 5 -year survival rate was extremely low among these patients (16). Our results indicated that of 47,911 lung cancer patients who survived beyond 5 years, $19.1 \%(\mathrm{~N}=9,162)$ developed a second primary cancer. Surprisingly, a significantly lower occurrence of SPM was observed in patients who received radiation therapy by age- and PSM-adjusted competing risk 

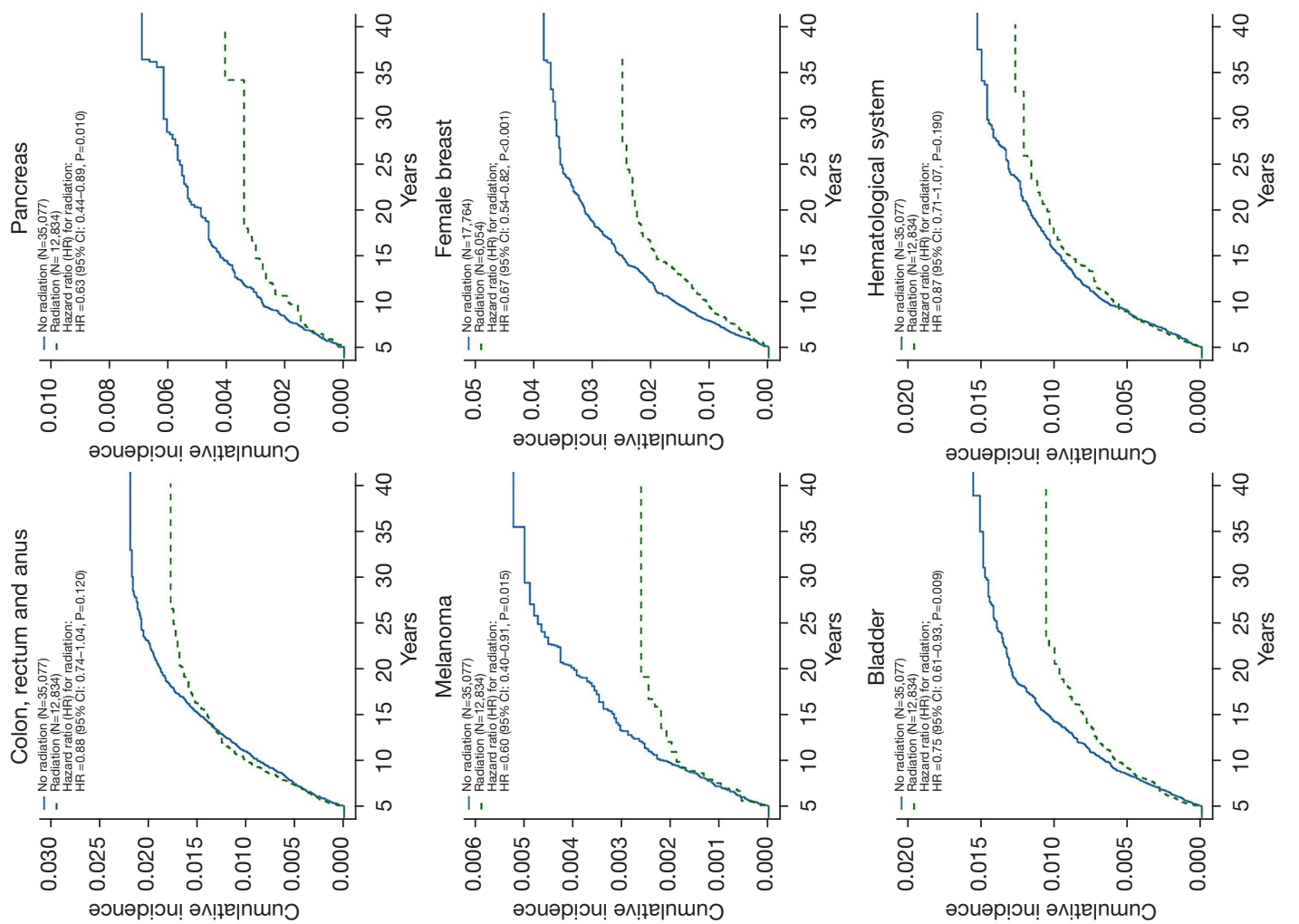

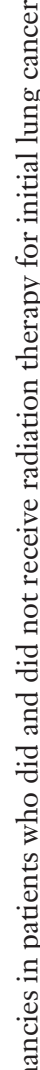
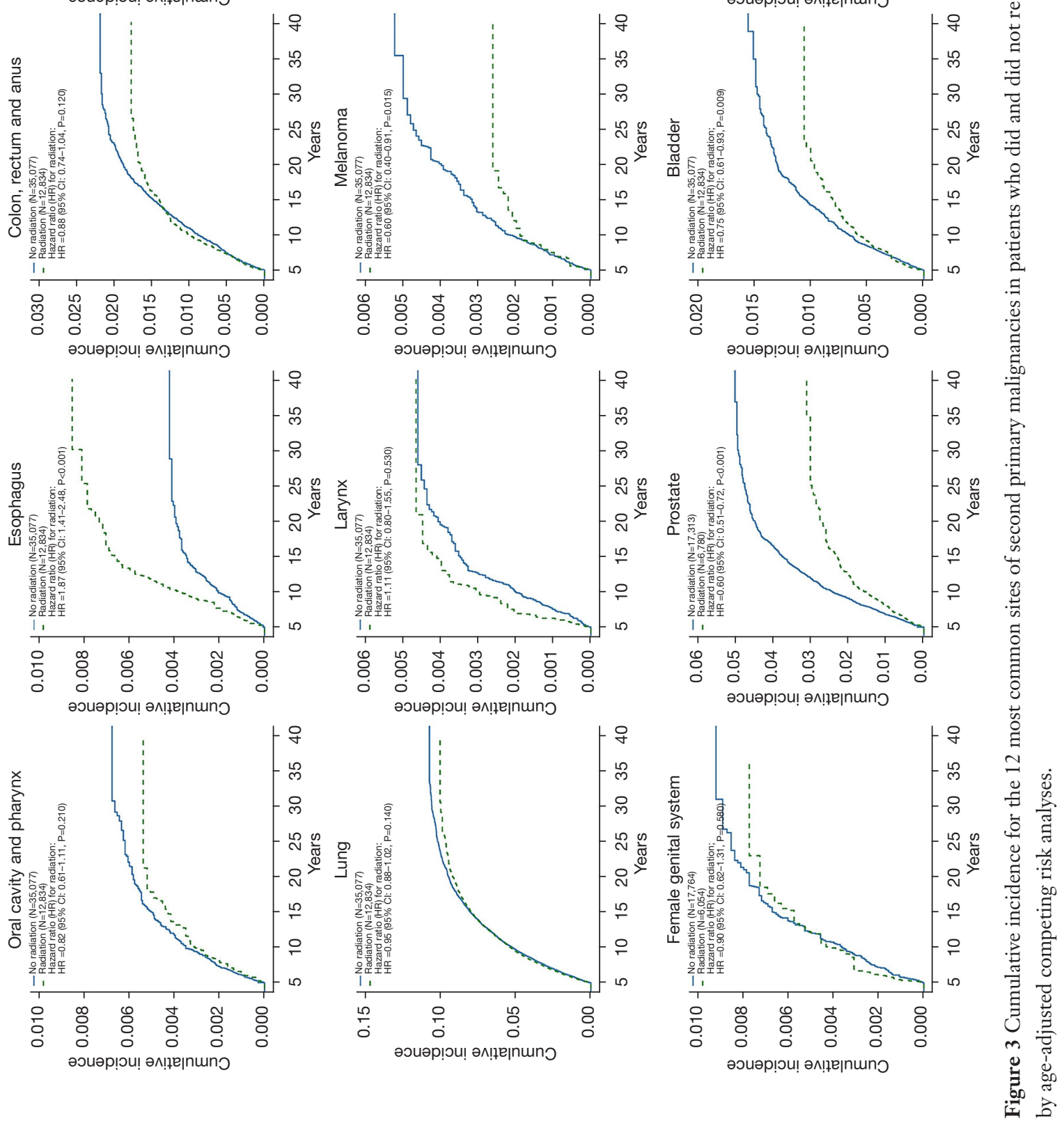


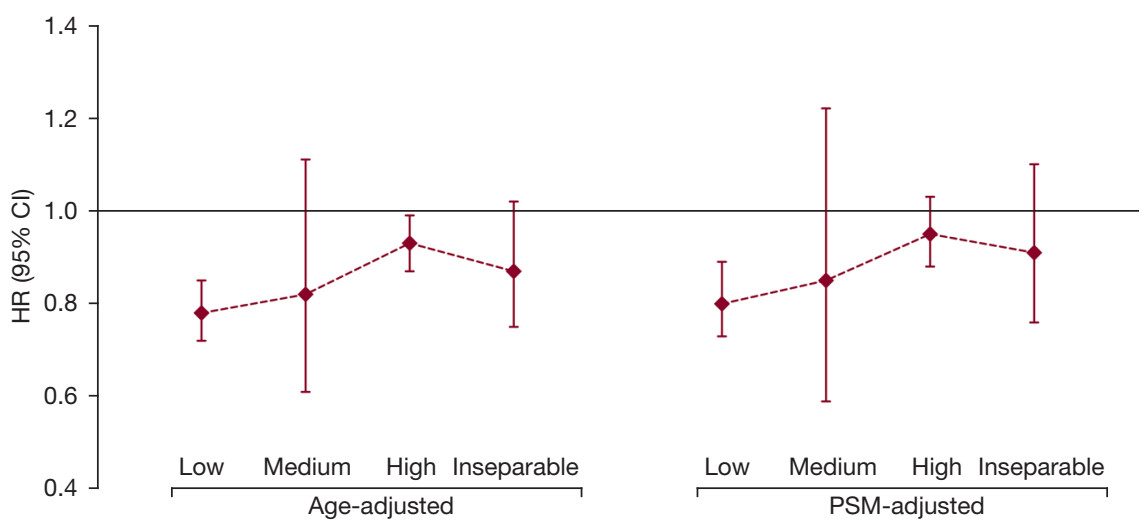

Figure 4 The risk for second primary malignancies at different dose-group sites in patients who did and did not receive radiation therapy for initial lung cancer by age-and propensity score matching (PSM)-adjusted competing risk analyses. Low-dose: hepatobiliary system; stomach; colon, rectum and anus; pancreas; female genital system; prostate; bladder; melanoma; nervous system; and kidney. Medium-dose: oral cavity and pharynx. High-dose: lung and bronchus; larynx; esophagus; female breast; and thyroid. Inseparable-dose: hematological system; miscellaneous; and other unknown tumors.

analyses than in those who did not. The risk of developing a specific second cancer varies dramatically. In our study, when calculating the risk of a specific second cancer in the radiation therapy group, other second cancers and death were regarded as competing events. There was a significant association between radiation therapy and the reduced incidence of developing a second primary melanoma as well as second primary cancers of female breast, prostate and thyroid in both age- and PSM-adjusted analyses. However, the risk of second primary esophageal cancer has increased dramatically.

Similar to our results, one previous study assessed the risk of second thoracic cancer following the diagnosis of the initial lung cancer and found that radiotherapy was significantly associated with an increased risk of second primary esophageal cancer when the latency was set as $\geq 5$ years, but no such significant difference was observed for second primary lung and breast cancers (20). The studies from both $\mathrm{Hu}$ et al. and Abdel-Rahman et al. reported that radiation therapy was significantly associated with a lower incidence of a metachronous second primary lung cancer within 5 years after the diagnosis of the initial lung cancer $(20,21)$. However, in contrast to our study, Berrington de Gonzalez et al. concluded that lung cancer patients receiving radiation therapy had an increased risk of developing SPM (9). This difference might be attributed to the fact that our study considered the cumulative risk related to the time effect by competing risk analyses. The event of death cannot be ignored when analyzing the risk of developing SPM, since patients who lived longer were more likely to suffer a second cancer. Both death and SPM should be regarded as time-to-event.

In general, patients who received radiation therapy were expected to be predisposed to developing SPM $(17,19)$. Consistent with other studies $(9,20)$, our study demonstrated an increased risk of second primary esophageal cancer in the radiation group (HR: 1.76, 95\% CI: 1.26-2.45, $\mathrm{P}<0.001$; Table 2). However, radiation therapy for initial lung cancer was associated with a significantly lower risk of second primary cancers in several sites, including skin (melanoma), female breast, prostate and thyroid gland. The conclusion from Berrington de Gonzalez et al. partly overlapped with ours, and they reported that the risk of second primary melanoma, prostate and thyroid cancers significantly decreased in NSCLC patients who received radiotherapy, but no significant effect was observed for second primary female breast cancer (9). Warschkow et al. demonstrated a significantly lower incidence of second primary prostate cancer in patients who received radiotherapy for rectal cancer (22). On the other hand, one previous study showed that radiation therapy for initial prostate cancer could increase the risk of developing SPM (23). According to studies by our group and others $(9,22)$, radiation for other organs or sites might be associated with a lower risk of second primary prostate cancer, which may deserve more studies in the future to confirm. Additionally, it was reported that radiation therapy for initial lung cancer increased the risk of breast cancer with a 5-year latency, but 
there was no significant difference with a latency $\geq 5$ years, which was confirmed by studies from our group and others $(9,20)$. However, based on conventional viewpoints, long survival was associated with increased susceptibility to SPM. With the rapid development of systemic therapy for lung cancer, the prognosis has significantly improved, but the risk of developing SPM varies by the second site. According to our results, radiotherapy did not significantly increase the risk of second malignancy other than esophageal cancer. On the one hand, for other second malignancies, distant location and low susceptibility to radiation might play an important part in reducing the risk of SPM. On the other hand, the survival benefit of additional therapy has had an impact.

Lung cancer was the most common second malignancy, accounting for $42.7 \%(3,908 / 9,162)$ of all second cancer patients. Thakur et al. demonstrated a higher risk of developing a second lung cancer in patients with previously treated lung cancer (7). Although many studies also concluded that radiotherapy was significantly associated with an increased incidence of second lung cancer, longterm follow-up results did not support the association in studies by our group and others $(9,20,21)$.

Unfortunately, we cannot obtain detailed information about radiation dose and modality from the SEER database. To identify the effects of dose on the incidence of SPM, we divided all SPM patients into several subgroups based on the distance of SPM organs or sites from the lung $(9,11)$. It could be inferred that the protection from SPM attributable to radiation therapy decreased in the second cancer sites with higher radiation doses (Figure 4). However, radiation techniques have changed substantially in recent decades, and the radiation doses for lung cancer have been standardized. Thus, this hypothesis requires stronger evidence and should be further verified in future studies.

The SEER database provides a large amount of data on clinical characteristics, treatment and follow-up information that is required for studies on SPM. Nevertheless, potential limitations should be acknowledged in this study. Like any observational and retrospective study, data bias cannot be absolutely eliminated. There might be some confounding factors, such as baseline clinical and treatment characteristics. Thus, we tried to minimize the confounding effects by PSM-adjusted analyses. Meanwhile, the use of a 5 -year latency between initial lung cancer and SPM could also reduce the follow-up bias between treatment groups. However, certain patient characteristics, such as smoking history, physical conditions and treatment details, are unavailable in the SEER database, which might have introduced patient bias between the two groups of patients with and without radiation and could have resulted in either overestimation or underestimation of the risks related to radiotherapy. Presumably, lung cancer patients who received radiotherapy might be more likely to be in a worse condition and could not tolerate surgery, which was indicated by the higher risk of death in patients who underwent radiation therapy (HR: 1.47, 95\% CI: 1.43-1.51, $\mathrm{P}<0.001$; Figure 2). Although the recent technological development of stereotactic body radiation therapy (SBRT) has achieved encouraging survival outcomes in early-stage NSCLC patients $(4,5)$, surgery is generally thought to be the first choice for resectable NSCLC. Hence, the result that radiation therapy decreased the risk of developing SPM should be interpreted cautiously.

In conclusion, using competing risk analyses, this study first demonstrated that radiation therapy for initial lung cancer was not associated with a higher overall risk of developing SPM in the long term. In contrast, a significantly decreased risk of second primary cancers of skin (melanoma), female breast, prostate and thyroid gland was observed in patients who received radiation therapy. However, the risk of developing second primary esophageal cancer dramatically increased after radiation.

\section{Acknowledgments}

Funding: This study was supported by a grant from the Ministry of Science and Technology of the People's Republic of China (Grant Number 2016YFC0105207). This study was funded by the National Key Research and Development Plan, the Ministry of Science and Technology of the People's Republic of China [grant number 2017YFC1311004].

\section{Footnote}

Reporting Checklist: The authors have completed the STROBE reporting checklist. Available at https://dx.doi. org/10.21037/jtd-21-915

Conflicts of Interest: All authors have completed the ICMJE uniform disclosure form (available at https://dx.doi. org/10.21037/jtd-21-915). The authors have no conflicts of interest to declare.

Ethical Statement: The authors are accountable for all aspects of the work in ensuring that questions related 
to the accuracy or integrity of any part of the work are appropriately investigated and resolved. The study was conducted in accordance with the Declaration of Helsinki (as revised in 2013). The study was reviewed and approved by the ethics review board of Peking Union Medical College Hospital. This was a non-interventional study based on the publicly-available datasets of the SEER database and thus the patient informed consent was not required.

Open Access Statement: This is an Open Access article distributed in accordance with the Creative Commons Attribution-NonCommercial-NoDerivs 4.0 International License (CC BY-NC-ND 4.0), which permits the noncommercial replication and distribution of the article with the strict proviso that no changes or edits are made and the original work is properly cited (including links to both the formal publication through the relevant DOI and the license). See: https://creativecommons.org/licenses/by-nc-nd/4.0/.

\section{References}

1. Siegel RL, Miller KD, Jemal A. Cancer statistics, 2020. CA Cancer J Clin 2020;70:7-30.

2. Vinod SK, Hau E. Radiotherapy treatment for lung cancer: Current status and future directions. Respirology 2020;25 Suppl 2:61-71.

3. Delaney GP, Barton MB. Evidence-based estimates of the demand for radiotherapy. Clin Oncol (R Coll Radiol) 2015;27:70-6.

4. Onishi H, Shirato H, Nagata Y, et al. Stereotactic body radiotherapy (SBRT) for operable stage I non-small-cell lung cancer: can SBRT be comparable to surgery? Int J Radiat Oncol Biol Phys 2011;81:1352-8.

5. Tandberg DJ, Tong BC, Ackerson BG, et al. Surgery versus stereotactic body radiation therapy for stage I nonsmall cell lung cancer: A comprehensive review. Cancer 2018;124:667-78.

6. Liu L, de Vries E, Louwman M, et al. Prevalence of multiple malignancies in the Netherlands in 2007. Int J Cancer 2011;128:1659-67.

7. Thakur MK, Ruterbusch JJ, Schwartz AG, et al. Risk of Second Lung Cancer in Patients with Previously Treated Lung Cancer: Analysis of Surveillance, Epidemiology, and End Results (SEER) Data. J Thorac Oncol 2018;13:46-53.

8. Zhou H, Shen J, Zhang Y, et al. Risk of second primary malignancy after non-small cell lung cancer: a competing risk nomogram based on the SEER database. Ann Transl Med 2019;7:439.
9. Berrington de Gonzalez A, Curtis RE, Kry SF, et al. Proportion of second cancers attributable to radiotherapy treatment in adults: a cohort study in the US SEER cancer registries. Lancet Oncol 2011;12:353-60.

10. Preston DL, Ron E, Tokuoka S, et al. Solid cancer incidence in atomic bomb survivors: 1958-1998. Radiat Res 2007;168:1-64.

11. Stovall M, Weathers R, Kasper C, et al. Dose reconstruction for therapeutic and diagnostic radiation exposures: use in epidemiological studies. Radiat Res 2006; 166:141-57.

12. Fine JP, Gray RJ. A Proportional Hazards Model for the Subdistribution of a Competing Risk. Journal of the American Statistical Association 1999;94:496-509.

13. Gray RJ. A Class of K-Sample Tests for Comparing the Cumulative Incidence of a Competing Risk. Ann Stat 1988;16:1141-54.

14. Scrucca L, Santucci A, Aversa F. Competing risk analysis using R: an easy guide for clinicians. Bone Marrow Transplant 2007;40:381-7.

15. Walter JE, Heuvelmans MA, de Jong PA, et al. Occurrence and lung cancer probability of new solid nodules at incidence screening with low-dose CT: analysis of data from the randomised, controlled NELSON trial. Lancet Oncol 2016;17:907-16.

16. Goldstraw P, Chansky K, Crowley J, et al. The IASLC Lung Cancer Staging Project: Proposals for Revision of the TNM Stage Groupings in the Forthcoming (Eighth) Edition of the TNM Classification for Lung Cancer. J Thorac Oncol 2016;11:39-51.

17. Huang YJ, Huang TW, Lin FH, et al. Radiation Therapy for Invasive Breast Cancer Increases the Risk of Second Primary Lung Cancer: A Nationwide Population-Based Cohort Analysis. J Thorac Oncol 2017;12:782-90.

18. Kaufman EL, Jacobson JS, Hershman DL, et al. Effect of breast cancer radiotherapy and cigarette smoking on risk of second primary lung cancer. J Clin Oncol 2008;26:392-8

19. Schaapveld M, Visser O, Louwman MJ, et al. Risk of new primary nonbreast cancers after breast cancer treatment: a Dutch population-based study. J Clin Oncol 2008;26:1239-46.

20. Abdel-Rahman O, Cheung WY. Subsequent thoracic cancers among patients diagnosed with lung cancer: a SEER database analysis. Curr Med Res Opin 2017;33:2009-17.

21. Hu ZG, Tian YF, Li WX, et al. Radiotherapy was associated with the lower incidence of metachronous 
second primary lung cancer. Sci Rep 2019;9:19283.

22. Warschkow R, Güller U, Cerny T, et al. Secondary malignancies after rectal cancer resection with and without radiation therapy: A propensity-adjusted, population-based
SEER analysis. Radiother Oncol 2017;123:139-46.

23. Wallis CJ, Mahar AL, Choo R, et al. Second malignancies after radiotherapy for prostate cancer: systematic review and meta-analysis. BMJ 2016;352:1851.

Cite this article as: Han C, Wu Y, Kang K, Wang Z, Liu Z, Zhang F. Long-term radiation therapy-related risk of second primary malignancies in patients with lung cancer. J Thorac Dis 2021;13(10):5863-5874. doi: 10.21037/jtd-21-915 
Supplementary

Table S1 The risk for second primary malignancies after radiation therapy for initial lung cancer in patients stratified by clinical and therapeutic characteristics

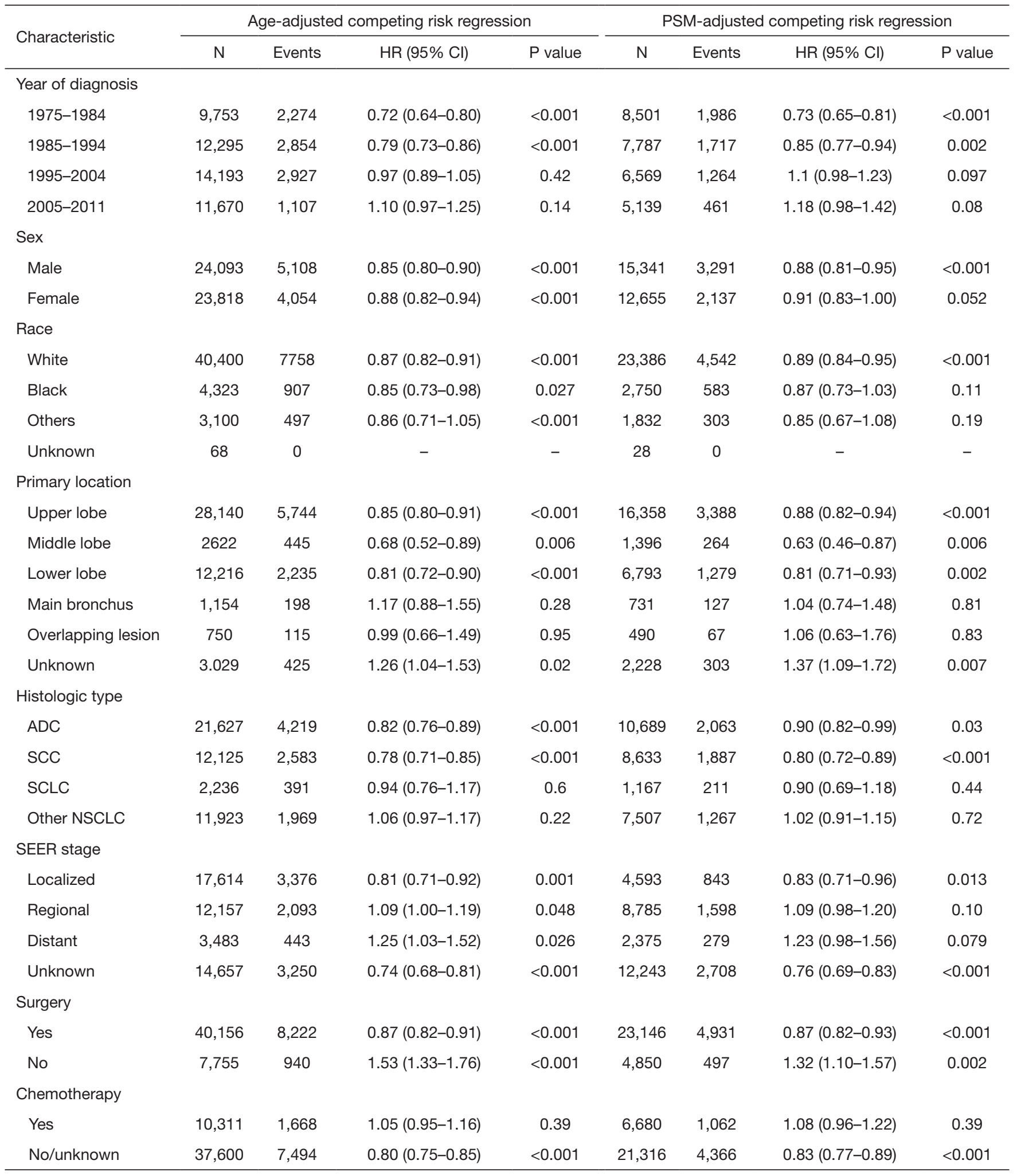


Table S2 The risk for second primary malignancies after radiation for initial lung cancer in different dose groups divided by the distance of second cancer from the lung

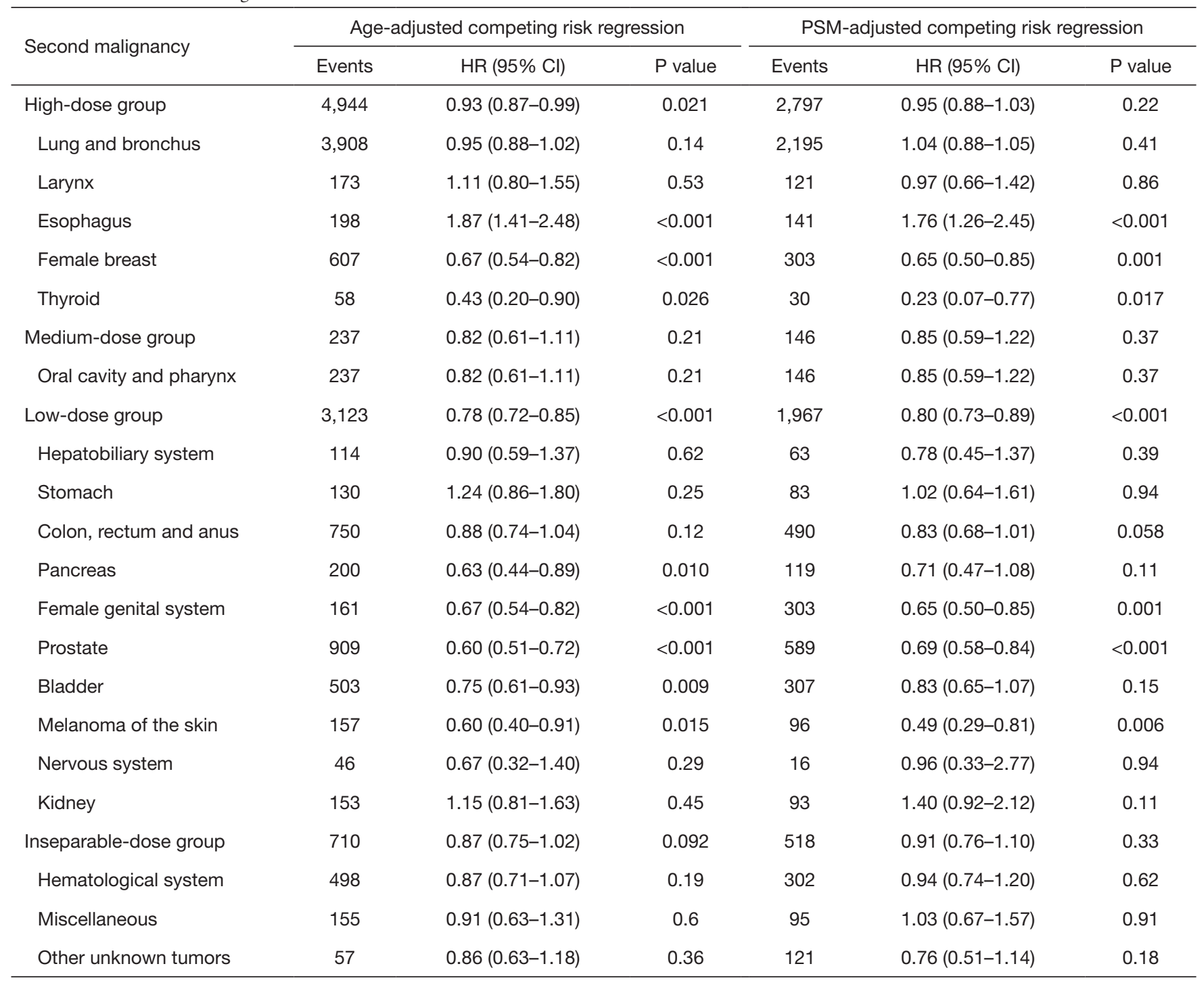

